\title{
A Study of Platelet Indices in Acute Myocardial Infarction: An Observational Study.
}

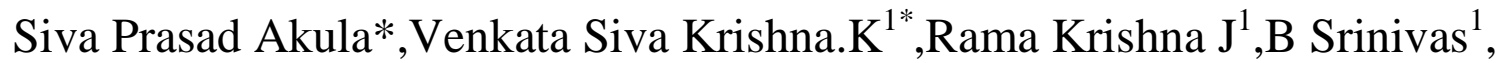 \\ SeshagiriraoDamera ${ }^{1}$. \\ ${ }^{I}$ Department Of Cardiology, Nizam's Institute Of Medical Sciences, Hyderabad. \\ *Corresponding Author: Venkata Siva Krishna.K
}

\begin{abstract}
Back ground: Platelets play an important role in the pathophysiology of Coronary Artery Disease(CAD). Naive platelets are larger in size and have more reactivity than older platelets. So the platelet indices including Mean Platelet Volume(MPV), Plateletcrit(Pct), and Platelet Distribution Width(PDW) may emerge a marker of CAD. The aim of the study is to study the role of platelet indices in patient with Acute Coronary Syndrome(ACS) and to see whether increase in platelet indices is associated with increased risk of ACS.

Materials and methods: In thepresent study 60 patients who presented to NIMS with Acute Coronary Syndromes(ACS) including Acute STEMI, NSTEMI were included. Platelet indices including Mean Platelet Volume $(M P V)$, Platelet Distribution Width $(P D W)$ and Platelet Crit $(P c t)$ were calculated in all these patients at the time of admission through automated analysers. These parameters were compared with 60 age and sex matched controls.

Results: The mean age of the study population is 56.3 years. In patients with ACS the mean platelet count was 2.35 lakhs, the mean MPV was 11.56fl, the mean Pct is $0.221 \%$, and mean PDW is 17.342. In the control population the mean MPV,PDW and Pct were 10.5fl,0.144\% and 15.214. In our study MPV did not show any statistically significant difference in patients with ACS. On the other hand there was a significant difference in Pct\& PDW in patients with ACS. Patients with ACS have higher values of Pct and PDW when compared to age matched controls. $(p=0.0001)$.

Conclusion: : Larger platelets are haemostatically more active and are a risk factor for developing coronary thrombosis and subsequent acute coronary event( Acute STEMI/NSTEMI ). Platelets with higher Pct and PDW were at higher risk of ACS. These patients can easily be identified during routine hematological analysis and could possibly benefit from preventive treatment.
\end{abstract}

Keywords: Acute Coronary Syndrome, Mean Platelet Volume, Platelet Distribution Width,PlateletCrit, Platelet reactivity.

\section{Introduction}

Platelet activation plays a central role in the transformation of atherosclerotic cardiovascular disease (CVD) into its potentially major adverse clinical events, such as ischemic stroke and myocardial infarction (MI) .Increased platelet activation may also represent the net patho-physiological effects of a number of CVD risk factors, such as smoking and raised cholesterol, thus representing a broad marker of CVD risk. Platelet activation leads to changes in platelet shape (increasingly spherical) with increased platelet swelling leading to an increase in platelet mass and volume. Traditional measures of platelet function/activation, such as the quantification of platelet derived metabolic parameters and the use of platelet aggregometry are technically difficult.Acute coronary syndrome result from acceleration of this chronic process characterized by rupture or fissuring of an unstable atherosclerotic plaque, accompanied by a cascade of platelet reactions resulting into thrombus formation.

Platelets play a crucial role in pathogenesis of atherosclerotic complications, contributing to thrombus formation or apposition after plaque rupture [10,11,12].After rupture of arteriosclerotic plaque in coronary arteries, platelets hyperactivity and local platelets activation have been suggested to play a causal role in prothrombotic events leading to MI[13].An increased platelet reactivity and shortened bleeding time are associated with increased platelet volume, therefore; platelet size has been considered to reflect platelet level of activity as the large platelets are more active than small platelets and they have a higher thrombotic potential due to high concentration of thromboxane A2.

\section{Patients And Methods}

The present study was done in Nizam's Institute of Medical Sciences, Hyderabad in the department of Cardiology and the blood samples were analysed by automated analysers in the pathology lab in the institute. 
All patients who were diagnosed to have Acute myocardial infarction (STEMI and NSTEMI) were included in the study. The window period was noted in all patients and the treatment done whether Medical Management (MM), Percutaneous Coronary Intervention (PCI)or Coronary Artery Bypass Surgery(CABG) is also noted.

Myocardial infarction is defined according to Third Universal Definition of $M I^{12}$ : the detection of a rise and/or fall of cardiac biomarker values, with at least one of the values being elevated (i.e., > 99th percentile upper reference limit, URL). The preferred cardiac biomarker of necrosis is the highly sensitive and specific cTn. In addition, at least one of the five following diagnostic criteria should be met:

1. Symptoms of ischemia

2. New (or presumably new) significant ST/T wave changes or left bundle-branch block (LBBB)

3. Development of pathological Q waves on ECG

4. Imaging evidence of new loss of viable myocardium or regional wall motion abnormality

5. Identification of intracoronary thrombus by angiography or autopsy.

Exclusion criteria: Patients with chest pain of $>6$ hours, Critically ill patients (ACS associated with renal failure, hepatic failure, myeloproliferative disorder or malignancy), patients having any platelet disorder as thrombocytopenia or thrombocytosis, patients with any bleeding or clotting disorder and patients on antiplatelet therapy.

Sample collection:Blood samples were taken from both groups of patients and controls. $2.5 \mathrm{ml}$ of blood were withdrawn in syringes by clean venipuncture; blood was dispersed in ethylene diaminetetraacetic acid (EDTA) tube. Blood samples from EDTA tube were put in automated analyzer for haematological analyses.

Statistical analysis: Statistical analysis was performed using SPSS software version 20 (La Jolla, CA, USA). Continuous variables were expressed as mean + standard deviation (SD) with range. Categorical variables were expressed as percentages. The difference in average of platelet indices values among ACS and non ACS cases were evaluated using student's t test. The level of significance was kept $95 \%$ for all statistical purposes.

\section{Results}

In our study there were 60 subjects with acute myocardial infarction were included. Of them 20 were female and M:F ratio is $2: 1$. The risk factor profile of the patients is shown in table 1 . These patients are compared with age and sex matched controls. As shown in table 1 there is no statistically significant difference in both the groups.

Table 1: Risk factor profile of the patients:

\begin{tabular}{|l|l|l|l|}
\hline Clinical profile & ACS $(\mathrm{N}=60)$ & NON ACS $(\mathrm{N}=60)$ & P VALUE \\
\hline Age + SD & $56.3+11.1$ & $48.9+10.3$ & 0.04 (Not significant) \\
\hline Hypertension & $48(77.36 \%)$ & $42(70 \%)$ & 0.43 (Not significant) \\
\hline Diabetes & $41(64.15 \%)$ & $36(60 \%)$ & 0.01 (Not significant) \\
\hline Alcoholics & $27(37.74 \%)$ & $30(50 \%)$ & 0.33 (Not significant) \\
\hline Smokers & $28(39.62 \%)$ & $20(33.33 \%)$ & 0.02 (Not significant) \\
\hline Dyslipidemia & $29(41.51 \%)$. & $26(43.33 \%)$ & 0.10 (Not significant) \\
\hline
\end{tabular}

Figure 1: Risk factor profile of the patients.

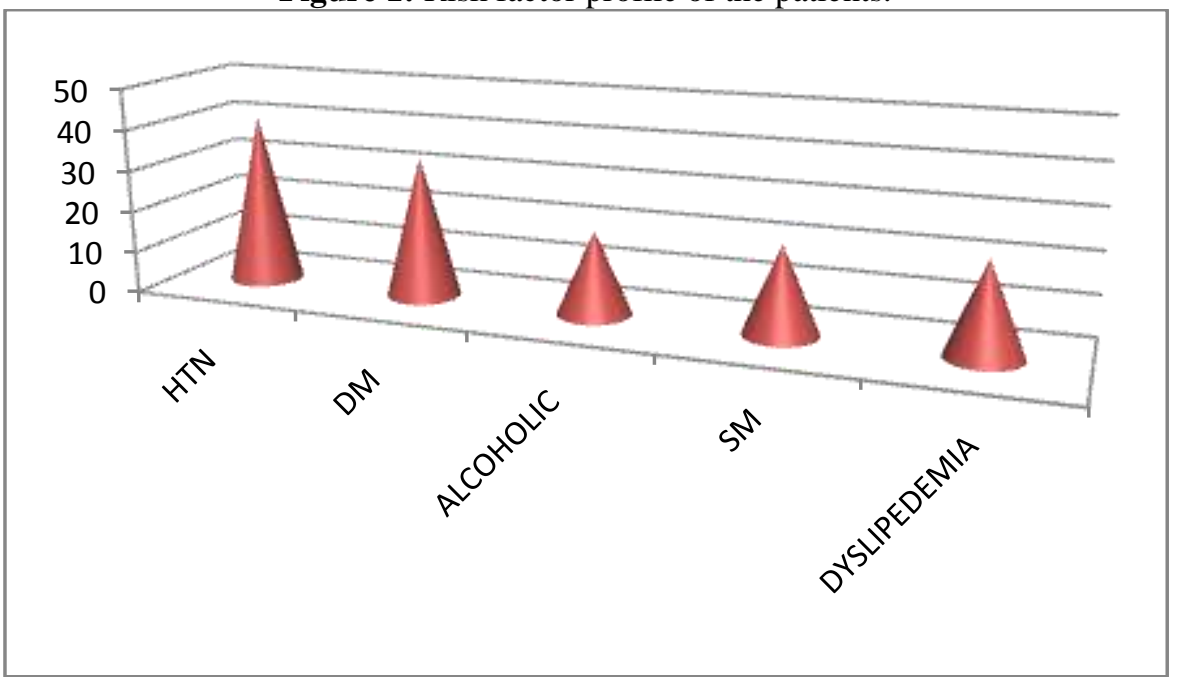


The mean age of the study population is $56.3 \pm 11.1$, the mean platelet count was 2.35 lakhs, the mean MPV was 11.56fl, the mean PCT is $0.221 \%$, and mean PDW is 17.342 .

Fig.1: Box plot of platelet count.

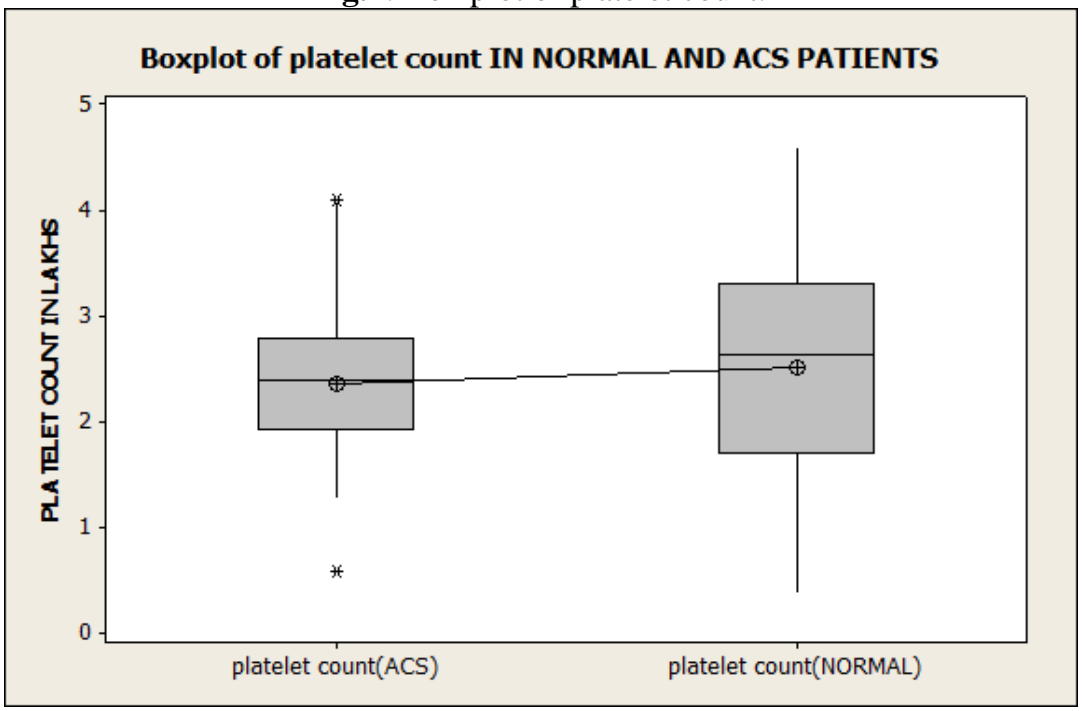

Box plot showed no significant difference in the platelet count in ACS and Normal patients and P value is not significant.

Table 2:Comparision of platelet indices in the two groups.

\begin{tabular}{|l|l|l|l|}
\hline & ACS & NON-ACS & P VALUE \\
\hline Platelet count & 2.389434 & 2.1139 & 0.112 \\
\hline MPV & 11.30755 & 10.5 & 0.04 \\
\hline Pct & 0.22166 & 0.144 & $<0.0001$ \\
\hline PDW & 17.34151 & 15.214 & $<0.0001$ \\
\hline
\end{tabular}

Fig 3 : Box plot if PDW in ACS patients and normal patients

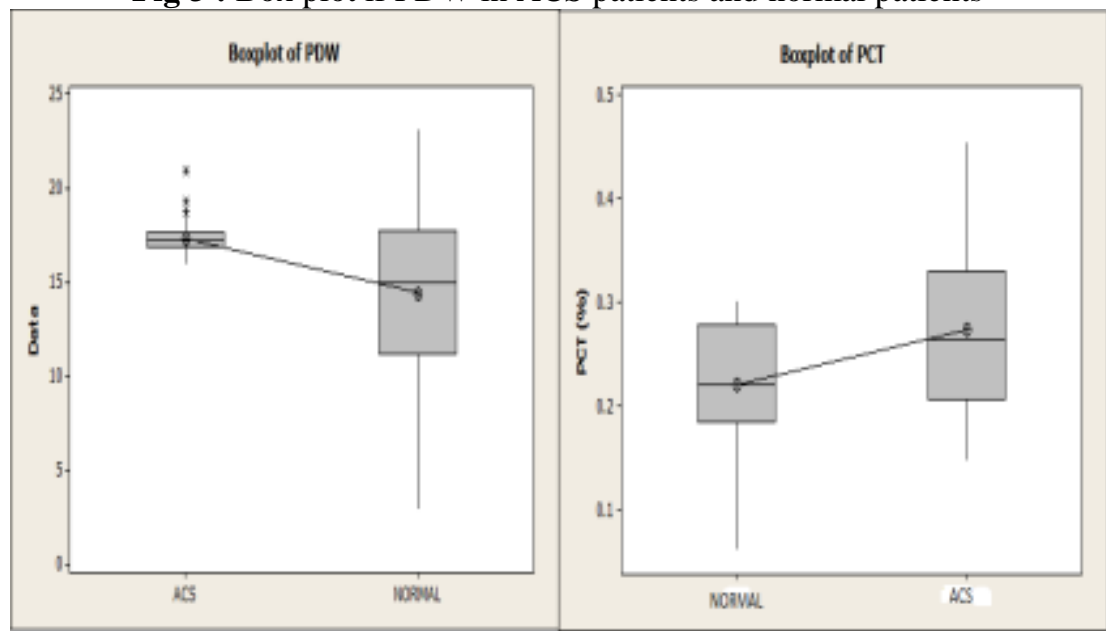

Patients with ACS were having a higher PDW and Pctwhen compared with normal persons. There is statisticallygross difference in Pctand PDW between normal and ACS patients with t values 4.58 and -4.05 respectively. The $\mathrm{p}$ value is statistically significant with $<0.0001$ for both Pct and PDW. In the present MPV did not show a statistically significant difference in ACS patients when compared to control group.

\section{Discussion}

Platelet activation leads to the formation of free arachidonic acid, which can be transformed into prostaglandins, such as thromboxane $\mathrm{A} 2$, one of the most potent vasoconstriction and platelet-aggregating substances, or into leukotrienes, which can amplify the acute inflammatory response. Platelet function and size correlate because larger platelets, produced from activated megakaryocyte in the bone marrow, are likely to be more reactive than normal platelets because large platelets contain more secretary granules and mitochondria and are known to be more active than small platelets (normal)(14). Consequently, larger and hyperactive 
platelets play a vital role in accelerating the formation and propagation of intracoronary thrombus, leading to the occurrence of acute thrombotic events. The difference in mean platelet indices between MI subject with STEMI and those NSTEMI(ECG change and enzyme positive) was statistically not significant similar to an Indian study that was done by Khandekar that found no association between the type of infarction and MPV.

Martin etal found that MPV was significantly higher in those patients who has MI $^{13,14}$. Van der Loo et al (Turkey) in their study suggested that platelet volume was an important biological variable to determine platelet reactivity.Nurcankiliclicamur et al studied mean platelet volume to be a predictive marker for acute myocardial infarction.Mathur et al found platelet counts to be significantly lower and MPV to be higher in patients with unstable angina pectoris.Butkiewicz et al showedthat there is no significant difference found between unstable angina pectoris and healthy control in terms of MPV. In view of these conflicting results the role of these indices is a matter of debate. MPVand PDW measurement, whichis a non-invasive and easy-toperform method,may be an useful tool for the follow-up, risk stratification and further studies are needed to confirm these findings. In our study there is higher Pct and PDW in ACS patients compared to Non ACS patients. MPV in the present study does not show significant difference. But still the other platelet indices showed significant difference.

\section{Conclusions}

1. Larger platelets are hemostatically more active and are a risk factor for developing coronary thrombosis, leading to myocardial infarction.

2. The findings of our study, Pct/PDW at admission are significantly higher inpatients with diagnosed ACS than in those with chest painof non-cardiac origin.

3. This finding can be a therapeutic target in the future.

4. Platelet indices can be used in risk stratification of the patients with ACS.

\section{References}

[1]. Boos CJ, Lip GY. Platelet activation and cardiovascular Outcomes in acute coronary syndromes. J ThrombHaemost 2006; 4: 25423.

[2]. Tsiara S, Elisaf M, Jagroop IA, Mikhailidis DP. Platelets as predictors of vascular risk: is there a practical index of platelet Activity. ClinApplThrombHemost 2003; $9: 177-90$.

[3]. Jesty J, Yin W, Perrotta P, Bluestein D. Platelet activation in a circulating flow loop: combined effects of shear stress and exposure time. Platelets 2003; $14: 143-9$.

[4]. Boos CJ, Lip GY. Assessment of mean platelet volume in coronary artery disease. Thromb Res 2006; 120:11-3.

[5]. Ruf A, Patscheke H. Flow cytometric detection of activated platelets: comparison of determining shape change, fibrinogen binding, and P-selectin expression. SeminThrombHemost 1995; 21:146-

[6]. Gurney D, Lip GY, Blann AD. Reliable plasma marker of platelet activation. Am J Hematol 2002; 70:139-44.

[7]. Kamath S, Blann AD, Lip GY .Platelet activation: assessment and quantification. Eur Heart J 2001; 22:1561-71.

[8]. Trip MD, Cats VK, van Capelle FJL, et al. Platelet hyperreactivity and prognosis in survivors of myocardial infarction. New Engl J Med 1990; 322: 1549-54.

[9]. Harrison L F.Principle of Internal Medicine.19th edition.2012,P 12

[10]. Ross R. Atherosclerosis: an inflammatory disease. N Engl J Med 1999; 340:115-26).

[11]. Jakubowski JA, Adler B, Thompson CB, etal.Influence of platelet volume on the ability ofprostacyclin to inhibit platelet aggregation and the release reaction. J Lab Clin Med 1985; 105: 271-6.

[12]. Mann D. Braunwald's heart disease. 10th ed.

[13]. Martin JF, Plumb J, Kilbey RS, Kishk YT. Changes in volume and density of platelets in myocardial infarction. BMJ. 1983; 287: 456-459.

[14]. Martin JF, Bath PM, Burr ML: Influence of platelet size on outcome after myocardial infarction. Lancet, 1991; 338(8780): 14091411 .

[15]. NurcanKilicli-Camur, R.Demirtunc, C.Konuralp,A. Eskiser, Y Basaran: Med Sci Monit,2005; 11(8): CR387-392. 